\title{
Ion Induced Magnetization Reorientation in Co/Pt Multilayers for Patterned Media
}

\author{
D. Weller, J.E.E. Baglin, A.J. Kellock, K.A. Hannibal, M.F. Toney, G. Kusinski ${ }^{(1)}$, S. Lang, L. Folks, M.E. Best and B.D. Terris \\ IBM Almaden Research Center, 650 Harry Rd., San Jose, CA 95120 \\ ${ }^{(1)}$ NCEM, Materials Science Division, Lawrence Berkeley National Laboratory, Berkeley, CA 94720
}

\begin{abstract}
$\mathrm{Co} / \mathrm{Pt}$ multilayer films with perpendicular magnetic anisotropy and large out-of-plane coercivities of 3.9 - 8.5 kOe have been found to undergo a spin reorientation transition from out-of-plane to in-plane upon irradiation with $700 \mathrm{keV}$ nitrogen ions. X-ray reflectivity experiments show that the multilayer structure gets progressively disrupted with increasing ion dose, providing direct evidence for local atomic displacements at the $\mathrm{Co} / \mathrm{Pt}$ interfaces. This effectively destroys the magnetic interface anisotropy, which was varied by about a factor of two, between $\mathrm{K}_{\mathrm{S}} \cong 0.4 \mathrm{erg} / \mathrm{cm}^{2}$ and $\mathrm{K}_{\mathrm{S}} \cong 0.85 \mathrm{erg} / \mathrm{cm}^{2}$ for two particular films. The dose required to initiate spinreorientation, $6 \cdot 10^{14} \mathrm{~N}^{+} / \mathrm{cm}^{2}$ and $1.5 \cdot 10^{15} \mathrm{~N}^{+} / \mathrm{cm}^{2}$, respectively, scales with $\mathrm{K}_{\mathrm{S}}$. It is roughly equal to the number of Co interface atoms per unit interface area contributing to $\mathrm{K}_{\mathrm{S}}$.
\end{abstract}

\section{Introduction}

Ion beam patterning of magnetic thin films through suitable stencil masks has been suggested recently as a prospective path towards patterned, ultrahigh-density magnetic recording media [1,2]. Co/Pt multilayers, in particular, are ideally suited for this application, since bombardment with a suitable dose of energetic ions may induce interface mixing [3], thereby triggering a spin-reorientation transition from easy axis out-ofplane to easy axis in-plane [1]. $\mathrm{He}^{+}$irradiation at $30 \mathrm{keV}$ ion energy and $10^{16}$ ions $/ \mathrm{cm}^{2}$ dose was reported by Chappert et al. [1] to effectively reduce the anisotropy, coercivity and Curie temperature in $\mathrm{Pt} / \mathrm{Co} / \mathrm{Pt}$ sandwich and multilayer structures. The mechanism leading to the observed modification of magnetic properties is not well understood, but it is likely due to local atomic relaxation and alloy formation [1]. Here we provide direct evidence of interfacial mixing, based upon Xray reflectivity and magneto-optic data.

\section{Experimental}

For the specific experiments discussed here, $\mathrm{Co} / \mathrm{Pt}$ multilayers with representative structure $[\mathrm{Si}$-substrate $/ 40 \mathrm{~nm}$ $\mathrm{SiN}_{\mathrm{x}} / 20 \mathrm{~nm}$ Pt buffer/10\%(0.3-0.4 nm Co/1 nm Pt) $/ 2 \mathrm{~nm} \mathrm{Pt}$ cap layer] were fabricated. The $\mathrm{SiN}_{\mathrm{x}}$ films were dc-magnetron sputtered and the $\mathrm{SiN}_{\mathrm{x}}$-coated substrates were heated to about $400^{\circ} \mathrm{C}$ before electron beam evaporation of the multilayers at growth temperatures $\mathrm{T}_{\mathrm{G}} \cong 210-230^{\circ} \mathrm{C}$. Growth rates were $\sim 0.05 \mathrm{~nm} / \mathrm{s}$ for Co and $\mathrm{Pt}$ and the pressure during evaporation was in the low $10^{-7}$ Torr range. The resulting films were characterized using Rutherford backscattering spectrometry (RBS) and transmission electron microscopy (TEM with Philips CM200FEG) as well as X-ray diffraction (XRD) for compositional and structural analysis, respectively. Nitrogen ion bombardment of $8 \% 8 \mathrm{~mm}^{2}$ areas at $700 \mathrm{keV}$ energy was performed using a rastered beam from a NEC 3UH Pelletron accelerator. Energy and ion species were selected to resemble the density of ionization conditions used in [1]. Magnetic measurements were performed using polar and transverse Kerr hysteresis measurements and torque magnetometry.

\section{Results and Discussion}

Fig. 1 shows a plan view TEM micrograph and an electron diffraction pattern of a $20 \mathrm{nmPt} / 10 \%(0.3 \mathrm{~nm} \mathrm{Co} / 1 \mathrm{~nm} \mathrm{Pt}) / 1 \mathrm{~nm}$ multilayer grown at $250^{\circ} \mathrm{C}$ onto a $\mathrm{SiN}_{\mathrm{x}}$ membrane.

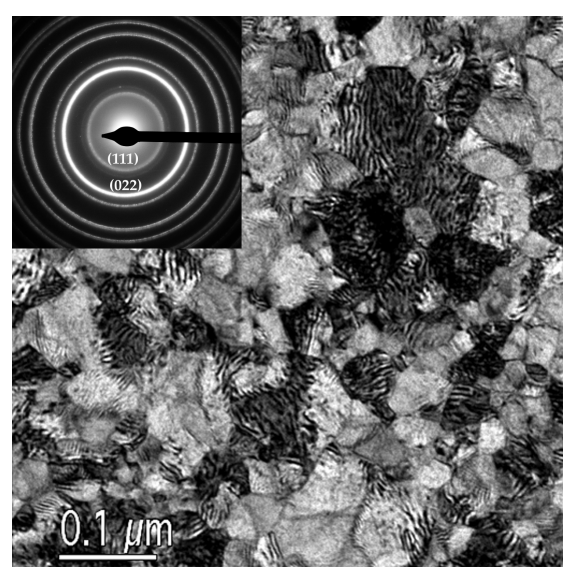

Fig. 1: Transmission electron micrograph and selected area diffraction pattern (SADP) of a representative $\mathrm{Co} / \mathrm{Pt}$ multilayer (see text).

The film is (111) textured and shows evenly distributed grains varying in diameter between $30-80 \mathrm{~nm}$. The polycrystalline structure, with [111] $\mathrm{Pt}$ and Co directions out of plane, is verified by selected area diffraction patterns (SADP) (see inset to Fig.1). The fine structure visible on some of the grains is attributed to Moiré fringes caused by small lattice mismatch 
and rotation between Co and Pt planes. CoPt(111) rocking curve widths of $\sim 9.3^{\circ}$ were found in XRD.

Table 1: Selected properties of two Co/Pt multilayers grown on $\mathrm{SiN}_{\mathrm{x}}$ coated $\mathrm{Si}(001)$ substrates with nominally identical structures $(10 \mathrm{x}(0.3 \mathrm{nmCo} / 1.0 \mathrm{nmPt}))$ grown at $230^{\circ} \mathrm{C}$ (sample I) and $210^{\circ} \mathrm{C}$ (sample II). The anisotropy constants are per Co volume. The large difference in $K_{u}, K_{S}$ and $H_{C}$ is attributed to structural differences and possibly slightly different $t_{C o}$.

\begin{tabular}{|l|l|l|l|}
\hline sample & $\mathrm{H}_{\mathrm{C}}{ }^{0}(\mathrm{Oe})$ & $\mathrm{K}_{\mathrm{u}}{ }^{0}\left(\mathrm{erg} / \mathrm{cm}^{3}\right)$ & $\mathrm{K}_{\mathrm{S}}{ }^{0}\left(\mathrm{erg} / \mathrm{cm}^{2}\right)$ \\
\hline $\mathrm{I}$ & 8500 & $6.7 \cdot 10^{7}$ & $0.84-0.86$ \\
\hline $\mathrm{II}$ & 3950 & $3.7 \cdot 10^{7}$ & $0.39-0.41$ \\
\hline
\end{tabular}

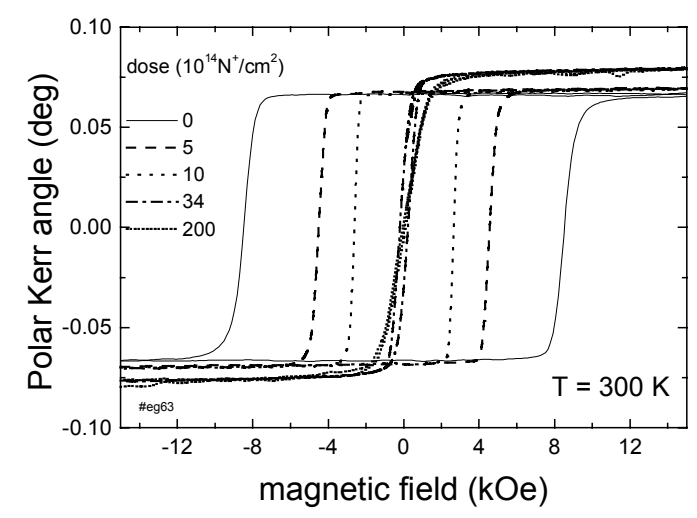

Fig. 2: Polar Kerr hysteresis loops of a Co/Pt multilayer subjected to $\mathrm{N}^{+}$ion irradiation at various doses.

Two similar samples, I and II, as listed in table 1 and grown on $\operatorname{SiN}_{\mathrm{x}}$ coated $\mathrm{Si}(001)$ substrates, are discussed in the following. Fig. 2 shows perpendicular hysteresis loops of sample I, before and after irradiation with $700 \mathrm{keV} \mathrm{N} \mathrm{N}^{+}$ions at various doses. The initial coercivity is $H_{C}{ }^{0}=8500 \mathrm{Oe}$, similar to that in [4] and [5] and ten times larger than in [1]. This is attributed to the large intrinsic anisotropy and the granularity of the present films (see Fig. 1). As in 1], strong sensitivity of $H_{C}$ to dose is observed. Doses as low as $10^{14} \mathrm{~N}^{+} / \mathrm{cm}^{2}$ reduce $H_{C}$, without, however, affecting the rectangular loop shape. Substantial decrease in the perpendicular remanence is observed for doses above $\sim 10^{15} \mathrm{~N}^{+} / \mathrm{cm}^{2}$. It is interesting to note that this corresponds roughly to the Co interface atomic density of $\sim 1.5 \cdot 10^{15}$ atoms per $\mathrm{cm}^{2}$ in these films. Note also, that the polar Kerr angle increases with dose. This is different from the results of Chappert et al. [1], where they reported a transition to paramagnetic behavior accompanied by a reduction of the Curie temperature, $T_{C}$, as well as the room temperature Kerr effect upon He-irradiation of $\mathrm{Pt} / \mathrm{Co} / \mathrm{Pt}$ sandwich structures. The present films have Curie temperatures, $T_{C}>300^{\circ} \mathrm{C}$, and do not show evidence of the onset of paramagnetism at room temperature. The enhancement of the Kerr rotation by about $15 \%$, as evident in Fig. 2, is characteristic of these and a variety of other films.
$\mathrm{Co} / \mathrm{Pt}$ multilayers deposited on different substrates. For example, $\mathrm{Si} / \mathrm{SiN}_{\mathrm{x}}, \mathrm{SiO}_{2}$ or $\mathrm{Al}_{2} \mathrm{O}_{3}$, all showed similar Kerr enhancements of the order of $10-15 \%$, independent of ion induced reductions of the reflectivity. The effect is therefore intrinsic. It may be interpreted as a direct consequence of interface mixing of $\mathrm{Co}$ and $\mathrm{Pt}$ atoms, resulting in local "alloying". Such resulting CoPt alloys are well known for their enhanced magneto-oplif effects because of stronger Pt$5 \mathrm{~d}$ electronic contributions [4].

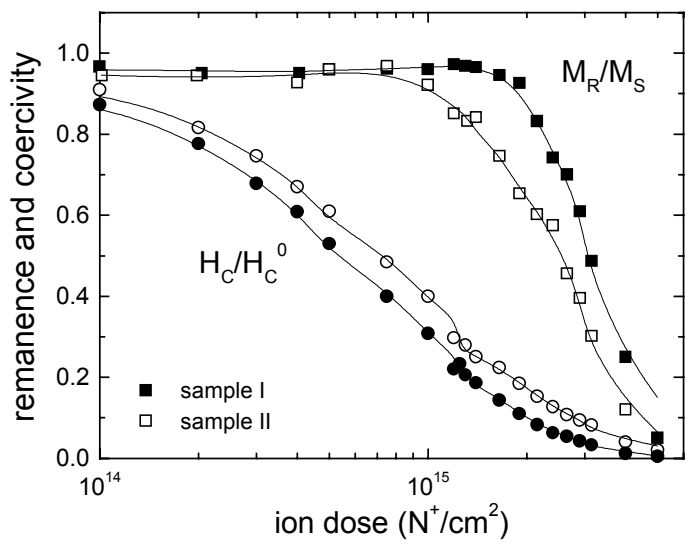

Fig.3: Perpendicular remanence $\left(\mathrm{M}_{\mathrm{r}} / \mathrm{M}_{\mathrm{S}}\right)$ and normalized coercivity $\left(\mathrm{H}_{\mathrm{C}} / \mathrm{H}_{\mathrm{C}}{ }^{0}\right)$ as function of $\mathrm{N}^{+}$ion dose for two films with vastly different initial coercivity and anisotropy.

Fig. 3 shows room temperature perpendicular coercivity and remanence data as a function of $\mathrm{N}^{+}$ion dose in the range $10^{14}-5 \cdot 10^{15} \mathrm{~N}^{+} / \mathrm{cm}^{2}$ for sample I and II. Both films show strong uniaxial perpendicular anisotropy, deduced from torque magnetometry using a $45^{\circ}$ method (see table 1 . such large anisotropies are not uncommon for $\mathrm{Co} / \mathrm{Pt}$ films $[5,6]$ and are attributed to an interface contribution, $K_{S}$, arising from the orbital overlap of Co-3d and Pt-5d wavefunctions. After irradiation with $>3-5 \cdot 10^{15} \mathrm{~N}^{+}$ions $/ \mathrm{cm}^{2}, K_{S}$ is quenched and the

$$
K_{u}=K_{V}+2 \pi M_{S}^{2}+\frac{2 \cdot K_{S}}{t_{C o}}
$$

total measured anisotropy energy has dropped to $\mathrm{K}=5 \cdot 10^{5}$ $\mathrm{erg} / \mathrm{cm}^{3}$. Commonly, one writes

with $\mathrm{K}_{V}$ a total volume anisotropy contribution, $2 \pi M_{S}^{2}$ the demagnetization energy and $t_{C o}$ the Co layer thickness. We estimate $K_{S}^{I}=0.84-0.86 \mathrm{erg} / \mathrm{cm}^{2}$ and $K_{S}^{I I}=0.39-0.41 \mathrm{erg} / \mathrm{cm}^{2}$, for samples I and II, respectively. The spread in $K_{S}$ results from assumptions about $K_{V}$, which is not well known for 1.5 monolayer thick Co films. Measurements on sputtered and MBE grown (111 textured Co/Pt multilayers indicate $K_{V}=1.2$ $-2.6 \cdot 10^{6} \mathrm{erg} / \mathrm{cm}^{3}[6]$. The demagnetization term is estimated to be $2 \pi M_{S}^{2}=1.6 \cdot 10^{7} \mathrm{erg} / \mathrm{cm}^{3}$ assuming a polarization enhanced $M_{S} \cong 1600 \mathrm{emu} / \mathrm{cm}^{3}$ per Co volume [6]. 
The normalized coercivities decrease gradually with dose, almost identical to the trend observed in $K_{u}$ (some data indicated in Fig. 3). We therefore conclude that the loss in coercivity is mainly interface anisotropy driven. The transition from out-of-plane to in-plane easy axis orientation occurs over a dose range of $\Delta \cong 3-4 \cdot 10^{15} \mathrm{~N}^{+} / \mathrm{cm}^{2}$ in both cases, which reflects the statistical nature of the bombardment. The onset of the transition, on the other hand, is sample dependent and correlates with the measured surface anisotropy $K_{S}$. It is determined by the balance of (positive) out-of-plane anisotropy energy and (negative) demagnetization energy contributions in eq.(1).

We estimate the atomic density of Co atoms per interface area to be $\mathrm{N}_{\mathrm{i}}^{\mathrm{Co}} \cong 1.5 \cdot 10^{15} \mathrm{~cm}^{-2}$. A fraction of about $85 \%$ (sample I) and $40 \%$ (sample II) of these Co interface atoms, i.e. $\mathrm{N}_{\mathrm{I}}^{\mathrm{Co}} \cong 1.3 \cdot 10^{15} \mathrm{~cm}^{-2}$ and $\mathrm{N}_{\mathrm{II}}{ }^{\mathrm{Co}} \cong 6 \cdot 10^{14} \mathrm{~cm}^{-2}$, respectively, should contribute to $K_{S}$. This assumes a maximal, intrinsic interface anisotropy value of $K_{S} \cong 1 \mathrm{erg} / \mathrm{cm}^{2}(100 \%)$ (see e.g. results found in highly perfected MBE Co/Pt films [6). Interestingly, these are exactly the doses needed to trigger spin reorientation in the present samples.

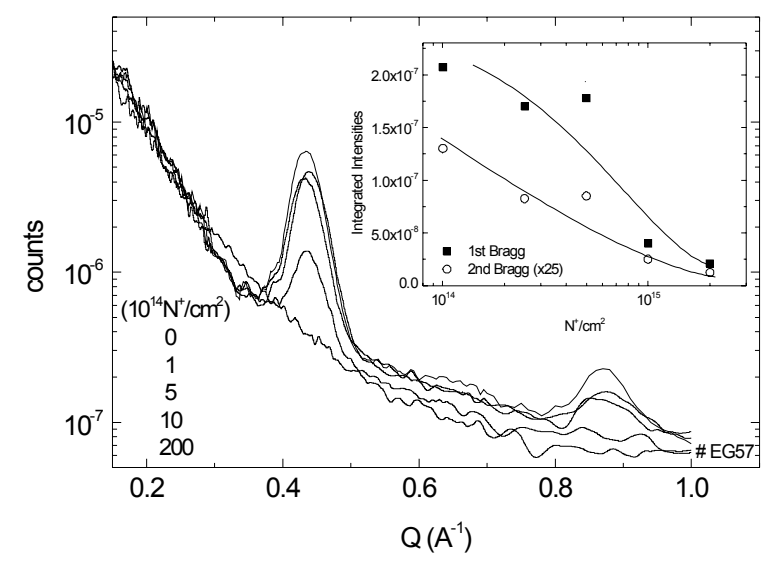

Fig.4: Diffuse or off specular X-ray reflectivity spectra and integrated Bragg peak intensities for different ion doses.

Fig. 4 shows diffuse or off specular X-ray reflectivity [7] data of a $20 \mathrm{~nm} \mathrm{Pt} / 10 \%(0.3 \mathrm{~nm} \mathrm{Co} / 1 \mathrm{~nm} \mathrm{Pt})$ multilayer for different ion doses. The diffuse reflectivity is the scattered intensity obtained just off the specular reflection condition [7], and the presence of the 'Bragg' peaks in these data show that the multilayer roughness is conformal over long lateral length scales. The trend in coercivity is closely related to that in the integrated intensity of the first and second Bragg peak in the diffuse reflectivity indicated in the inset to Fig. 4. This drop in intensity shows directly that there is a significant interfacial intermixing on short lateral length scales with increasing ion dose and this destroys the perpendicular anisotropy, due to the disruption of abrupt $\mathrm{Co} / \mathrm{Pt}$ interfaces. At the highest dose of $2 \cdot 10^{16} \mathrm{~N}^{+} / \mathrm{cm}^{2}$ in this experiments, the multilayer is completely converted into a $\mathrm{Co}_{\sim 25} \mathrm{Pt}_{\sim 75}$ alloy.
The present data indicate that a constant number of 3-4 impinging $\mathrm{N}^{+}$ions per surface atomic area is needed to rearrange one Co-Pt interface pair and remove their contribution to $K_{S}$. Essentially, all Co-Pt atomic pairs need to be displaced (randomized) to effectively remove the perpendicular anisotropy. This was found to hold for the number of bilayers varying between 2 and 20. Each bilayer evidently responds to the incident ion flux in identical fashion, as anticipated, since the energy and fluence of $700 \mathrm{keV} \mathrm{N}$ ions are negligibly degraded on the present thickness scale.

The mechanism leading to atom rearrangements is not obvious. A ballistic recoil mechanism, predicted by simple TRIM [8] simulations, would be far too sparse to explain the present results. It is reasonable to assume that thermodynamically preferred local cluster configurations of $\mathrm{Co}_{1-\mathrm{x}} \mathrm{Pt}_{\mathrm{x}}$ atoms, as suggested by Chappert et al. [1], are assisted to form without a large ballistic energy transfer. Such local relaxation of the interface order could possibly be stimulated by energy transferred from the ion via ionization and lattice phonons. To test this concept, a detailed model will be required, taking account of the threshold energies for displacement of specific $\mathrm{Co}$ and $\mathrm{Pt}$ atoms in the interface configuration.

D.W. and L.F. gratefully acknowledge the support by AMRI and DOD/DARPA through Grant No. MDA 972-97-1-003. S.L. acknowledges financial support from DAAD.

\section{References}

1 C. Chappert, H. Bernas, J. Ferré, V. Kottler, J. -P. Jamet, Y. Chen, E. Cambril, T. Devolder, F. Rousseaux, V. Mathet, H. Launois, "Planar Patterned Magnetic Media Obtained By Ion Irradiation”, Science 280, 1919 (1998);J. Ferré, C. Chappert, H. Bernas, J.-P. Jamet, P. Meyer, O. Kaitasov, S. Lemerle, V. Mathet, F. Rousseaux, H. Launois "Irradiation Induced Effects on Magnetic Properties of Pt/Co/Pt Ultrathin Films", J. Magn. Magn. Mater. 198-199, 191 (1999); T. Devolder, C. Chappert, Y. Chen, E. Cambril, H. Bernas, J.-P. Jamet, J. Ferré, "Sub 50 nm planar magnetic nanostructures fabricated by ion irradiation", Appl. Phys. Lett. 74, 3383 (1999).

2 B. Terris, L. Folks, D. Weller, J.E.E. Baglin, A.J. Kellock, H. Rothuizen, P. Vettiger, "Ion Beam Patterning of Magnetic Films Using Stencil Masks", Appl. Phys. Lett. 75, 403 (1999).

3 P.F. Carcia, S.I. Shah, W.B. Zeper, "Effect of energetic bombardment on the magnetic coercivity of sputtered Pt/Co thin-film multilayers", Appl. Phys. Lett. 56, 2345 (1990).

4 D. Weller, H. Brändle, C. Chappert, "Relationship between Kerr effect and perpendicular magnetic anisotropy in Co1-xPtx and $\mathrm{Co}_{1-\mathrm{x}} \mathrm{Pd}_{\mathrm{x}}$ alloys", J. Magn. Magn. Mater. 121, 461 (1993).

5 S. Shiomi, T. Nishimura, T. Kobayashi, M. Masuda, "Effect of Substrate Heating on Magnetic Properties of Co/Pt Multilayers on Pt Buffer Layers", Advanced Materials' 93 II/B: Information Storage Materials ed. M. Abe et al., Trans. Mag. Res. Soc. Jpn., Vol 15B, 1007 (1993).

6 D. Weller, R.F.C. Farrow, R.F. Marks, G.R. Harp, H. Notarys, G. Gorman, "Interface and volume anisotropy of MBE-grown Co/Pt(111), (110) and (001) and sputtered Co/Pt multilayers", Mat. Res. Soc. Symp. Proc. vol 313, 791 (1993).

7 V. Holy, U Pietsch, T Baumbach, "High-Resolution X-ray Scattering from Thin Films and Multilayers", Springer, Berlin, 1999.

8 TRIM (TRansport of Ions in Matter) Monte Carlo code simulating interaction of ions in matter: J.P. Biersack and L. Haggmark, Nuclear Instruments and Methods in Physics Research 174, 257 (1980). The TRIM software for PCs is available from J.F. Ziegler, jfz@us.ibm.com. 